\title{
SUPERCRITICAL THERMODYNAMICS OF SULFUR AND NITROGEN SPECIES
}

Project No. DE-FG22-91PC91287

Principal Investigator:

Initiation Date:

Period Covered by Report:
Prof. Charles A. Eckert

Georgia Institute of Technology

School of Chemical Engineering

(404) 853 - 9344

October 1, 1991

October 1, 1991 - December 31, 1991

\begin{abstract}
Significant opportunity exists for the application of supercritical fluid (SCF) technology to coal processing, both for pretreatment of high sulfur coals, as well as liquefaction and treatment of coal liquids. Supercritical fluids are attractive solvents for a variety of coal processing applications because of their unusual solvating and mass transfer properties. Solubility studies have been carried out for a number of model coal and coal-liquid compounds, primarily in pure supercritical fluids. We are extending this database of model coal compound equilibria using modern techniques that have the advantage of being much more rapid than traditional techniques. Cosolvent effects on solubility are being investigated over a variety of solvent properties. In addition, specific molecular interactions are being investigated through spectroscopic techniques. The resulting data is being used develop a chemical-physical equation of state (EOS) model of SCF solution with meaningful parameters. The equation of state will be used to predict solubility behavior, which will permit the design and tailoring of SCF cosolvent systems for specific coal processing applications.
\end{abstract}

\section{DISCLAIMER}

This report was prepared as an account of work sponsored by an agency of the United States Government. Neither the United States Government nor any agency thereof, nor any of their employees, makes any warranty, express or implied, or assumes any legal liability or responsibility for the accuracy, completeness, or usefulness of any information, apparatus, product, or process disclosed, or represents that its use would not infringe privately owned rights. Reference herein to any specific commercial product, process, or service by trade name, trademark, manufacturer, or otherwise does not necessarily constitute or imply its endorsement, recommendation, or favoring by the United States Government or any agency thereof. The views and opinions of authors expressed herein do not necessarily state or reflect those of the United States Government or any agency thereof.
MASTER

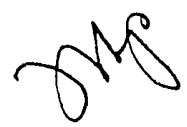




\section{OBJECTIVES}

The focus of this work is the application of supercritical fluid (SCF) solvents to the precombustion desulfurization and denitrogenization of coal, as well as to the liquefaction and subsequent processing of coal liquids. In particular, we focus on the design of solvent-cosolvent systems for treating nitrogen and sulfur heterocycles. A novel technique using supercritical fluid chromatography measures rapidly the cosolvent effect on solubility and is used to extend the existing database of solubility data. Further details of molecular interactions in supercritical fluids, including specific solvation and the "clustering phenomena" in the near critical region, are investigated using high-pressure spectroscopic experiments. Data obtained from these experiments will be used in the development of a chemical-physical equation of state that accounts for the unique solvation properties of supercritical fluid solutions using accurate and chemically-meaningful parameters.

Supercritical fluids (SCF) show potential for coal processing because they possess some unique properties. A SCF is quite dense, which gives a large capacity for solutes; it has high diffusivity and low viscosity, which makes it an ideal medium for efficient mass transfer; and the fluid is highly compressible, which yields large solubility changes with small changes in temperature and pressure. The addition of a small amount of cosolvent (usually less than 5\%) can dramatically enhance solubility. These phenomena suggest extraordinary selectivity possibilities. Therefore, we are proceeding with the measurement of solubilities in these novel fluids and fluid mixtures.

Solubility data exist for a wide variety of chemical compounds for pure solid solutes in several pure SCF's. The majority of these data are taken in SCF systems with a low critical point. Solubility data has recently been extended to include some heavier compounds typical of coal structures and solubilities in supercritical fluids with higher critical temperatures. These data have been correlated with a variety of cubic EOS's and semitheoretical expressions. However, in order to account for the effect of cosolvent on solubility and exploit potential selectivities in model coal compound systems, an EOS developed though a molecular approach is necessary.

Our ongoing studies of cosolvent effects both support the development of a chemical-physical EOS as well as extend the existing database of solubilities of model coal compounds in SCF solutions. The supercritical fluid chromatograpny technique permits rapid measurement of these effects. The solvent, cosolvent and solute are carefully chosen to gain maximum information on the relative importance of solute and cosolvent properties such as polarity, acidity, and basicity on phase equilibria. 
Direct spectroscopic measurements of the specific interactions which yield extreme solubility and cosolvent effects will also be used to understand the relative importance of specific interactions in SCF's. The spectroscopic phenomena investigated include charge-transfer complexing, hydrogen bonding, and dipole coupling. A high-pressure UV-vis spectrometer and a high-pressure fluorescence spectrometer are being used to probe these interactions. From the spectra information can be derived about the strength of both solute/solvent and solute/solute and solute/cosolvent forces and how they change with proximity to the critical point. Both the solubility and spectroscopy data are vital to the development of an equation of state model that will take into account both the physical and chemical furces that are important in SCF solutions. 


\section{PROGRESS THIS QUARTER}

\section{PHASE EOUILIBRIA AND COSOLVENT EFFECTS}

Under past DOE support, we compiled an extensive database of coal model compound solubilities. Although cosolvent modified SCF's show much promise in facilitating difficult separations, relatively little data exist on the effects of these modifiers. Both the magnitude and the importance of specific molecular interactions on cosolvents effects are yet unknown. Therefore, we are now extending this solubility database by using two novel techniques for measuring solubilities in supercritical fluid solutions containing cosolvents. Through carefully designed experiments, we are also working to delineate the effects of various specific interactions on model coal compounds solubilities in SCF solutions.

One reason that solubility and cosolvent data are scarce is that conventional techniques to measure solubilities are time consuming and require large amounts of solute. A chromatographic technique that we developed to measure solubilities and the effects of cosolvents on SCF solutions is attractive because chromatographic measurements are rapid, require little solute, and inherently separate impurities from the solute. The apparatus is shown in Figure 1.

With this technique, we are measuring solubilities and cosolvent effects based on the retention time of the solute in the column with the mobile phase as the SCF solution of interest versus the time spent in the column by an unretained solute. We define the cosolvent effect for a solute as the ratio of its solubility in the fluid mixture to the solubility in the pure fluid at the same temperature and pressure. We have recently demonstrated the viability of this technique (Ekart et al., 1992); the absolute solubilities measured using this technique compare favorably with literature data.

In order to investigate different types of interactions in SCF solutions, several different types of fluids, cosolvents, and solutes are being studied. The three fluids of interest are shown in Table 1 with their critical properties, dipole moments, and polarizabilities; note that the critical temperatures are similar. Ethane is a nonpolar fluid that has no specific interactions with any of the cosolvents or solutes. Because we are primarily interested in looking at cosolvent-solute interactions, the cosolvent studies will be done in ethane. Carbon dioxide is also nonpolar, but has a large quadrapole moment; in addition, carbon dioxide can participate in acid-base interactions, as it does when reacting with water to form carbonic acid. Fluoroform, $\mathrm{CHF}_{3}$, is polar and is a hydrogen bond donor.

Table 2 shows the various cosolvents that we are plan to study along with their Kamlet-Taft solvatochromic parameters (Kamlet and Taft, 1976; Karnlet et al., 
1977, 1983), dipole moments, and polarizabilities. The Kamlet-Taft format is useful for rating solvent strength by using linear solvation energy relationships to relate quantities such as reaction rates, UV absorption maxima, or chromatographic retention times to chemical characteristics of the solvent. A measurable quantity, $\mathrm{XYZ}$, is related to the parameters by

$$
X Y Z=X Y Z_{0}+a \alpha+b \beta+s \pi^{*}
$$

where $\mathrm{XYZ}_{\mathrm{o}}$ is an intercept, and $a, b$, an $s$, are solute-specific constants. The parameter $\alpha$ indicate the ability of the solvent to donate hydrogen bonds, $\beta$ denotes the ability to accept hydrogen bonds, and $\pi^{*}$ is a measure of solvent polarity/polarizability. It is important to remember that $\alpha$ and $\beta$ are only indications of hydrogen bonding ability and no more.

The cosolvents we will investigate include commonly used alcohols and acetone. In addition, chloroform and 2,2,2-trifluoroethanol (222-TFE) will be examined because of their ability to donate protons but not accept them. 1,1,1trichloroethane (111-TCE) is similar to chloroform, but is not a hydrogen bond donor. Conversely, triethylamine (TEA) will not donate protons but is an excellent acceptor; it also is a Lewis base that can take part in charge transfer complexes. Finally, 1,2-dibromoethane (12-DBE) was chosen because it neither accepts nor donates protons, but it has a large $\pi^{*}$ indicative of high polarity/polarizability. This choice of cosolvents allows us to study independently various effects, including hydrogen bonding, in SCF solutions.

Solubilities and cosolvent effects will be studied for various solutes (Table 3). These include nonpolar compounds such as anthracene and phenanthrene, alcohols like 9-hydroxyfluorene and the phenolic 2-naphthol, and amines such as carbazole, with a pyrollic nitrogen, and acridine, with a pyridinic nitrogen. To study the effect of specific constituents, we will also look at series of compounds such as fluorene, fluorenone, dibenzofuran, dibenzothiophene, carbazole and 9-hydroxyfluorene. In addition to nitrogen-containing compounds, sulfur compounds like thianthrene and dibenzothiophene will be studied, as cosolvent effects on sulfur compounds are also of interest to coal processing. Because we are currently using a UV detector, all of the solutes are UV active. Initial screening has shown that the solubilities of these solutes in these systems falls within an acceptable experimental range; the retention times of these solutes is significant.

Formulation of an experimental plan to delineate cosolvent effects based on chemical interactions is an integral part of our continued development of an equation of state with chemically-meaningful parameters. 


\section{SPECIFIC INTERACTIONS THROUGH SPECTROSCOPY}

An important modelling aspect of SCF solution behavior is the solvent structure about a solute molecule. We are currently studying local composition effects using 7-azaindole, which forms an exciplex with alcohols at low concentrations. Systems of indoles or substituted indole "solutes" in SCF's containing alcohol or water cosolvents are promising systems to probe local composition effects. The solvent dependent nature of their spectral features has been reviewed and the fluorescence kinetics examined (Tamaki, 1983). 7-Azaindole contains both a pyrrolic nitrogen and a pyridine-like nitrogen and is therefore representative of the heterocyclic compounds typically found in coal. Also, while alcohols are commonly used as SCF cosolvents, they characteristically cause quenching of fluorescence. Therefore, only specific systems allow for the study of intermolecular interactions in systems with alcohol cosolvents using fluorescence spectroscopy.

7-azaindole is a more sensitive than the naphthols we have previously studied (Tomasko et al., 1991) in the respect that one can monitor two independent interactions with the cosolvent. First, the tautomer of azaindole resulting from double proton transfer can be observed as a broad structureless emission red-shifted from the monomer by $120-160 \mathrm{~nm}$. It is proposed that the tautomer is formed from as a cyclic hydrogen bond complex in the ground state and requires one alcohol molecule per azaindole molecule. (Ingham and El-Bayoumi, 1970; Avouris et al., 1976). See Figure 2.

An exciplex emission arising from an excited state alcohol-azaindole complex can also be observed as a distinct red-shifted peak $(20-60 \mathrm{~nm})$ similar in shape and overlapping with the monomer fluorescence band. This alcohol and 7-azaindole exciplex has been studied thoroughly in liquids (Collins, 1983). Through kinetic analysis, the exciplex was found to result from a single alcohol complexing with a single azaindole molecule, as shown in Figure 3.

We have studied 7-azaindole in supercritical $\mathrm{CO}_{2}$ with $0.5,1.0,2.0$, and 4.5 mole percent methanol as a cosolvent. Figure 4 shows fluorescence spectra at all concentrations of cosolvent and at approximately the same density $(18 \mathrm{~mol} / \mathrm{l})$ The fluorescence intensities do not necessarily correspond to alcohol concentration due to lamp intensity. Two features are of note: first, the successive addition of alcohol shows a clear transition from monomer emission in pure SCF to exciplex emission in the mixture; second, even at $4.5 \%$ alcohol no proton transfer occurred. These observations require further analysis. 


\section{PLANS FOR NEXT QUARTER}

We have outlined an experimental plan to investigate cosolvent effects using a chromatographic technique. The solvents, cosolvents, and solutes have been carefully chosen to gain maximum understanding of the importance of specific chemical interactions on cosolvent effects. The solutes, many containing sulfur and nitrogen, have been screened to insure the feasibility of obtaining cosolvent data from their retention times. Next quarter, we plan to measure cosolvent effects on solubility on these systems.

7-azaindole is being used as a probe of solvent structure around a solute in SCF cosolvent systems. Fluorescence spectra indicate an exciplex emission without tautomer emission in alcohol cosolvent systems. The interpretation of these observations requires further study, which we plan to continue next quarter.

We will continue testing and improving our new chemical-physical equation of state based upon our solubility, cosolvent, and spectroscopic data.

\section{SUMMARY}

Supercritical fluids (SCF) show potential for a variety of coal processing applications. Application of SCF technology to these processes is limited by a dearth of information on the phase equilibria of coal compounds in SCF solutions and cosolvent effects on these solubilities. We are taking a molecular-based approach to developing and improving a chemical-physical equation of state with chemicallymeaningful parameters. The goal of this work is the ability to tailor SCF cosolvent systems to specific coal processing applications through knowledge of the chemical interactions which are significant to the SCF system.

Future work will include further investigations of cosolvent effects using supercritical fluid chromatography, fluorescence spectroscopy, and UV absorption. This information and the database of solubility measurements will be used in the further development of an equation of state model to predict solubilities for the design of coal treatment processes. 


\section{REFERENCES}

Avouris, P.; Yang, L.L.; El-Bayoumi, M.A., "Excited State Interactions of 7-Azaindole with Alcohol and Water," Photochem. and Photobiol., 1976, 24, 211.

Collins, S.T., "Exciplex Formation in Alcohol and Water Solutions of 7-Azaindole," J. Phys. Chem., 1983, 87, 3202.

Dewar, M.J.S.; Stewart, J.J.P., "A New Procedure for Calculating Molecular Polarizabilities; Applications Using MNDO," Chem. Phys. Lett.,1984, 111, 416.

Ekart, M.P., Bennett, K.L.,; Eckert, C.A., "A Chromatographic Technique to Study the Effects of Specific Interactions in Supercritical Fluid Solutions," submitted to ACS Sym. Ser., 1992.

Ingham, K.C.; El-Bayoumi, M.A., "Photoinduced Double Proton Transfer in a Model Hydrogen Bonded Base Pair. Effects of Temperature and Deuterium Substitution.", J. Am. Chem. Soc.,1974, 96(6), 1674.

Kamlet, M.J.; Taft, R.W., "The Solvatochromic Comparison Method. 1. the $\beta$-Scale of Solvent Hydrogen-Bond Acceptor (HBA) Basicities," J. Am. Chem. Soc., $1976,98,377$.

Kamlet, M.J.; Taft, R.W., "The Solvatochromic Comparison Method. 6. The $\pi^{*}$ Scale of Solvent Polarities," J. Am. Chem. Soc., 1977, 99, 6027.

Kamlet, M.J.; Abboud, J.L.; Abraham, M.H.;Taft, R.W., "Linear Solvation Energy Relationships. 23. A Comprehensive Collection of the Solvatochromic Parameters, $\pi^{*}, \alpha, \beta$ and Some Methods for Simplifying the Generalized Solvatochromic Equation," J. Org. Chem., 1983, 48, 2877.

Landolt-Börnstein, Zahlenwerte und Funktionen, 6th ed., Vol.1, Part 3, Springer-verlat, Berling, p. 510, (1951).

Reid, R.C.; Prausnitz, J.M.; Poling, B.E., The Properties of Gases \& Liquids, 3rd ed., MoGraw-Hill, New York, 1977.

Tamaki, T., "Steady-State Kinetic Studies on the Exciplex Formation between Indoles and n-Butyl Alcohol," J. Phys. Chem., 1983, 87, 2383. 
Tomasko, D.L.; Knutson, B.L.; Eckert, C.A.; Haubrich, J.E.; Tolbert, L.M., "Fluorescence Investigation of Cosolvent/Solute Interactions in Supercritical Fluid Snlutions," Submitted 5/91 to ACS Symposium Series. 
Table 1. Supercritical fluids and their properties of interest

\begin{tabular}{|l|c|c|c|c|}
\hline Fluid & $T_{c}(K)^{\mathrm{a}}$ & $\mathrm{P}_{\mathrm{c}}(\mathrm{bar})^{\mathrm{a}}$ & $\mu(\mathrm{D})^{\mathrm{a}}$ & $\alpha\left(\mathrm{cm}^{3} \times 10^{-25}\right)$ \\
\hline Ethane & 305.4 & 48.8 & 0 & $44.7^{\mathrm{b}}$ \\
\hline Carbon Dioxide & 304.1 & 73.8 & 0 & $26.5^{\mathrm{c}}$ \\
\hline Fluoroform & 299.3 & 48.6 & 1.6 & $27.4^{\mathrm{b}}$ \\
\hline
\end{tabular}

Table 2. Cosolvents and their properties

\begin{tabular}{|l|c|c|c|c|c|}
\hline Cosolvent & $\pi^{\mathrm{d}}$ & $\alpha^{\mathrm{d}}$ & $\beta^{\mathrm{d}}$ & $\mu(\mathrm{D})^{\mathrm{a}}$ & $\alpha\left(\mathrm{cm}^{3} \times 10^{-25}\right)$ \\
\hline Methanol & 0.60 & 0.93 & 0.62 & 1.7 & $32.3^{\mathrm{c}}$ \\
\hline Ethanol & 0.54 & 0.83 & 0.77 & 1.7 & $48.6^{\mathrm{b}}$ \\
\hline 2-Propanol & 0.48 & 0.76 & 0.95 & 1.7 & $64.2^{\mathrm{b}}$ \\
\hline Acetone & 0.71 & 0.06 & 0.48 & 2.9 & $63.3^{\mathrm{c}}$ \\
\hline Chloroform & 0.58 & 0.44 & 0.00 & 1.1 & $82.3^{\mathrm{c}}$ \\
\hline 1,1,1-Trichloroethane & 0.49 & 0.00 & 0.10 & 1.7 & $103.6^{\mathrm{b}}$ \\
\hline 2,2,2-Trifluoroethanol & 0.73 & 1.51 & 0.00 & & $52.8^{\mathrm{b}}$ \\
\hline Triethylamine & 0.14 & 0.00 & 0.71 & 0.9 & $134.9^{\mathrm{b}}$ \\
\hline 1,2-Dibromoethane & 0.75 & 0.00 & 0.00 & 1.0 & $106.5^{\mathrm{b}}$ \\
\hline
\end{tabular}

${ }^{2}$ Reid et al. (1987)

bestimated from Dewar and Stewart (1984)

Candolt-Börnstein (1951)

${ }^{d}$ Kamlet et al. (1983) 


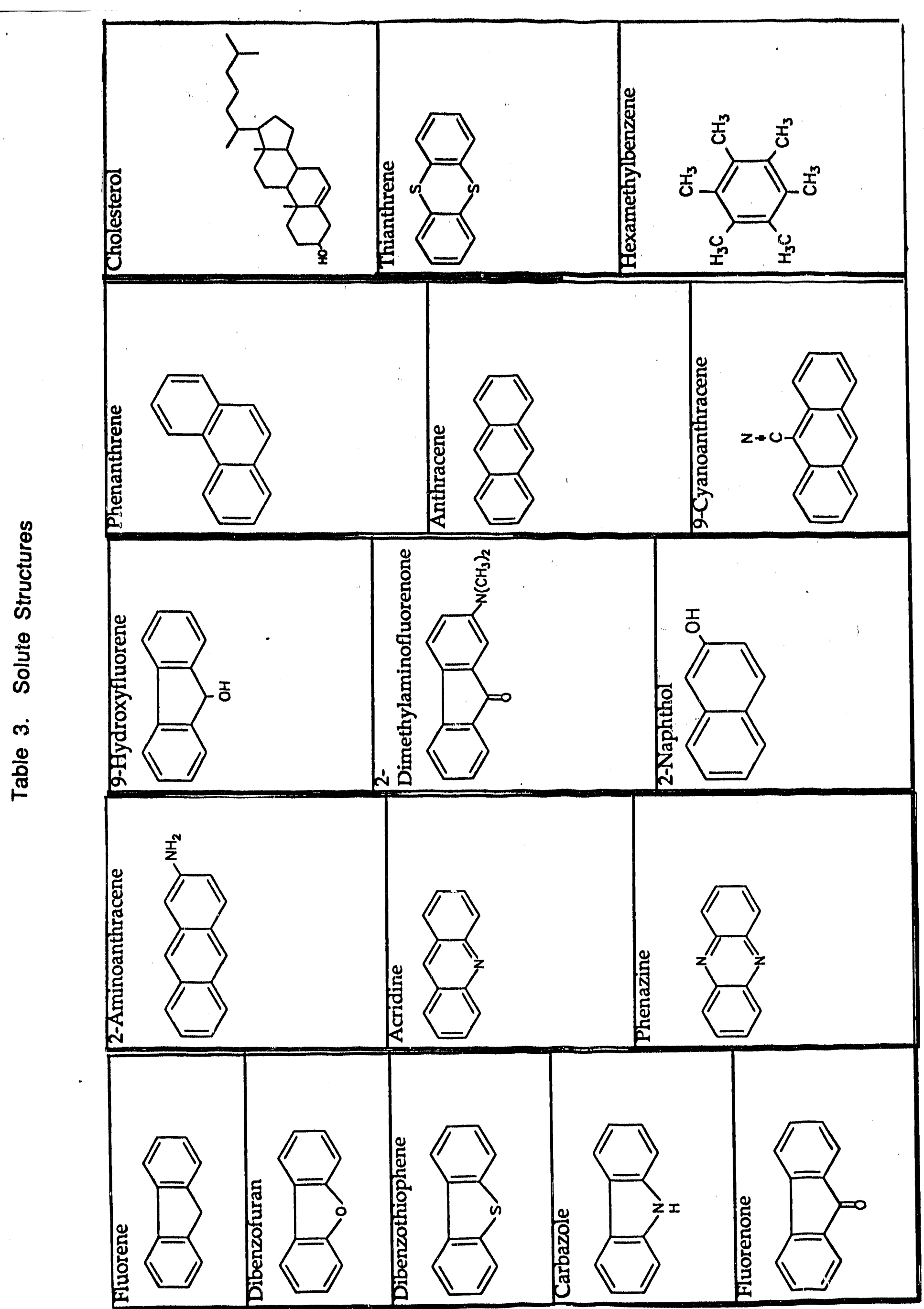




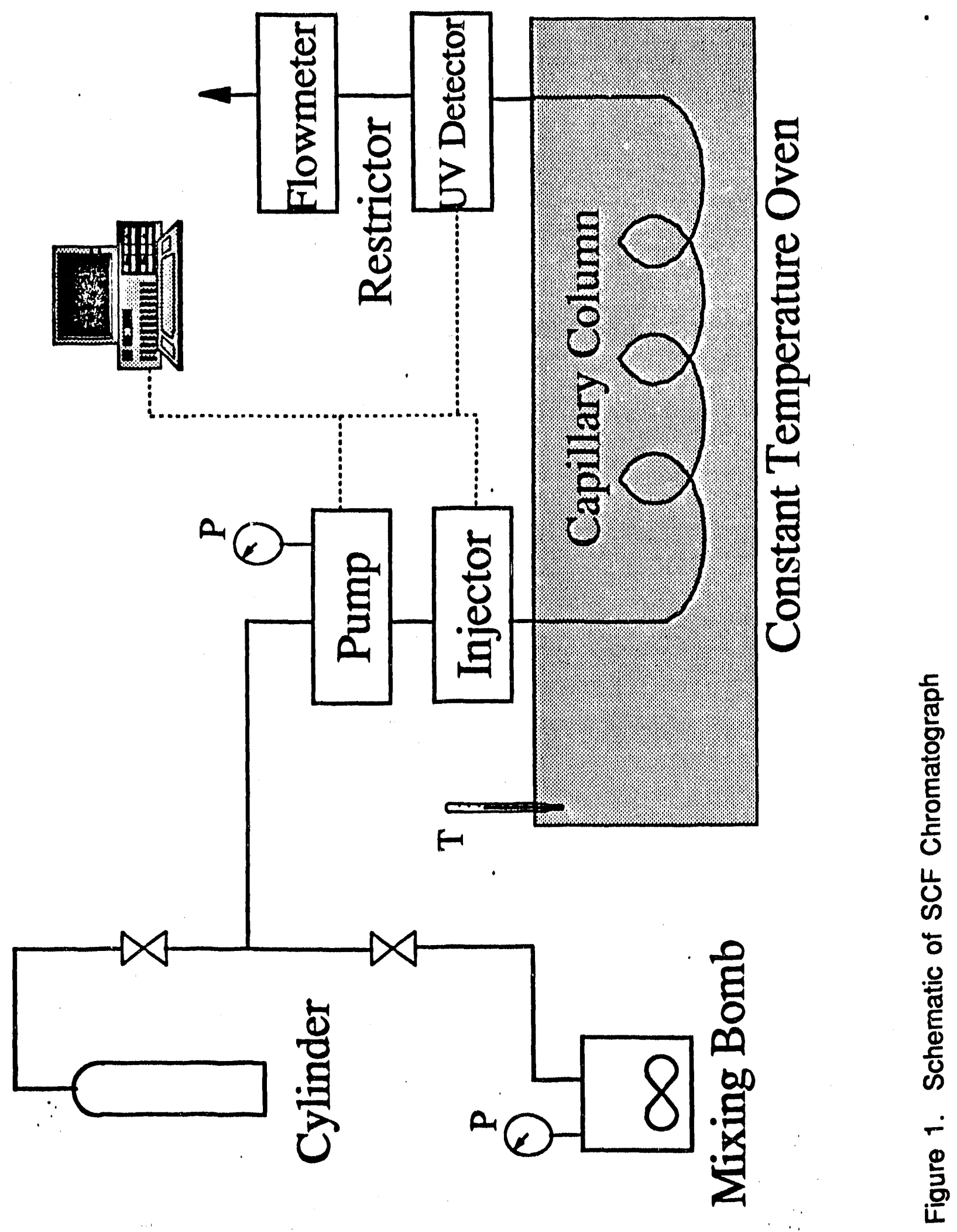



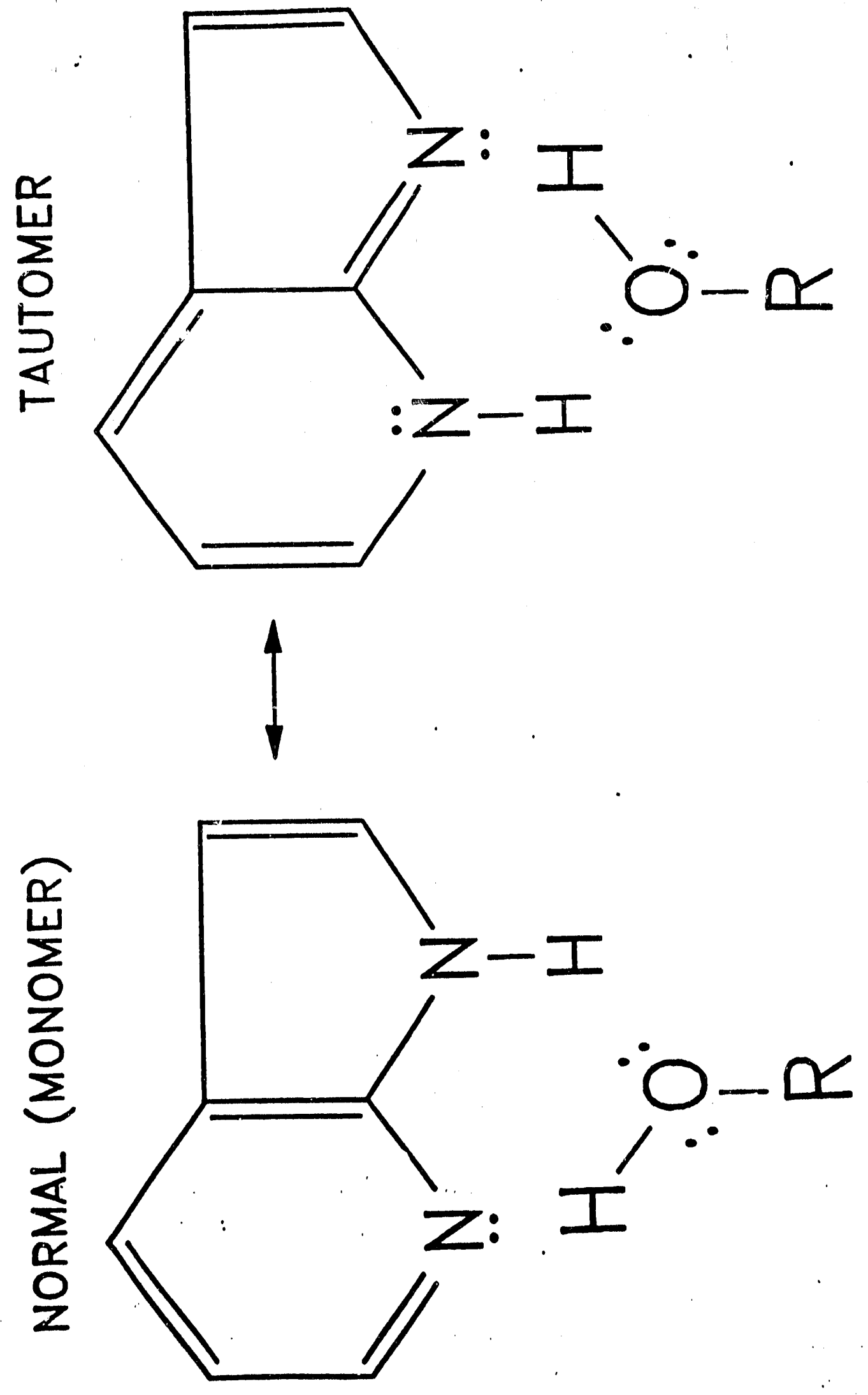

뭄

동

음

ऊ $\frac{0}{0}$

톤 엉

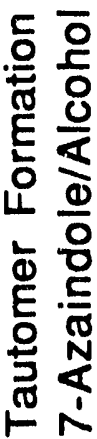

ำ 


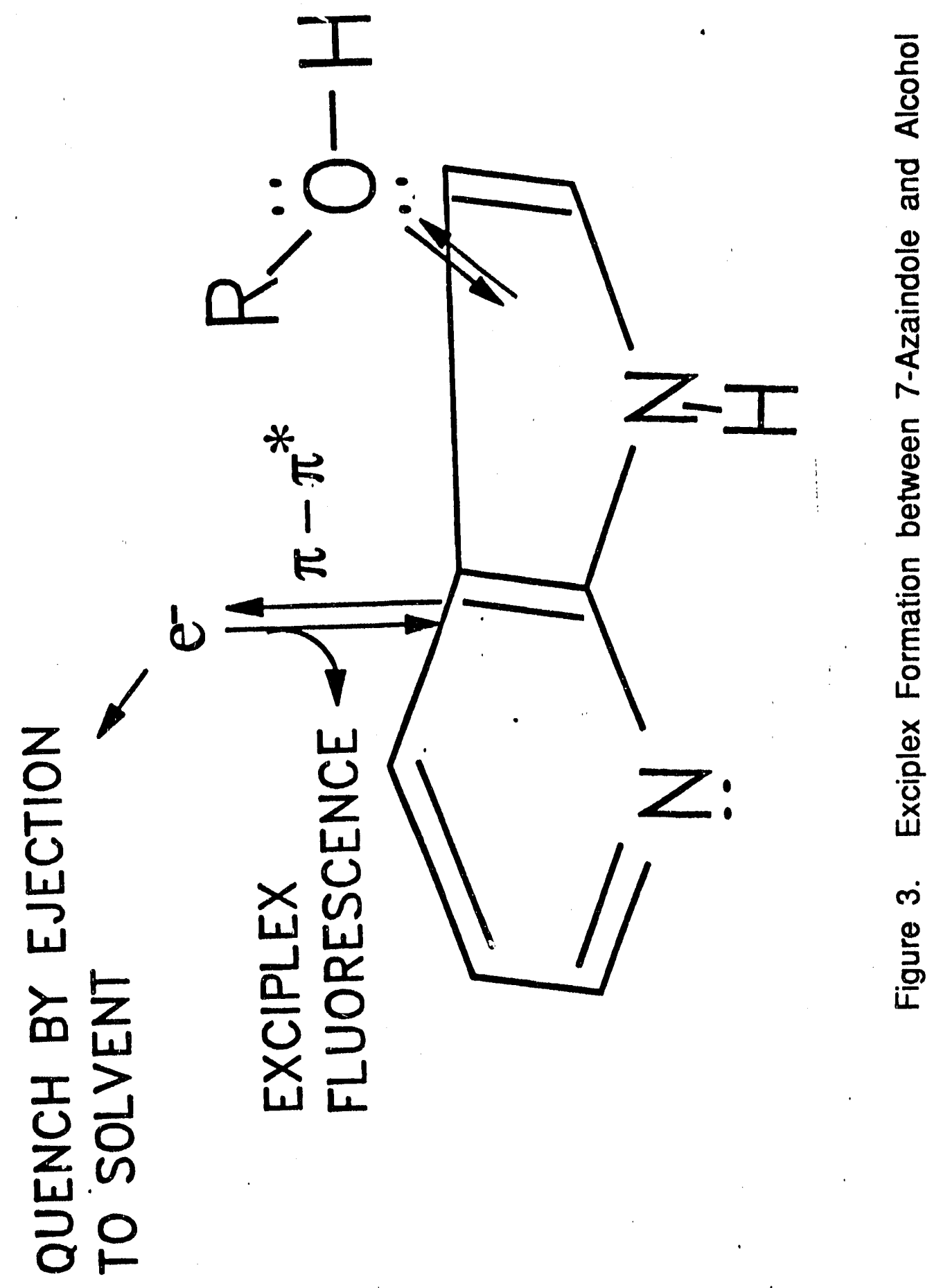




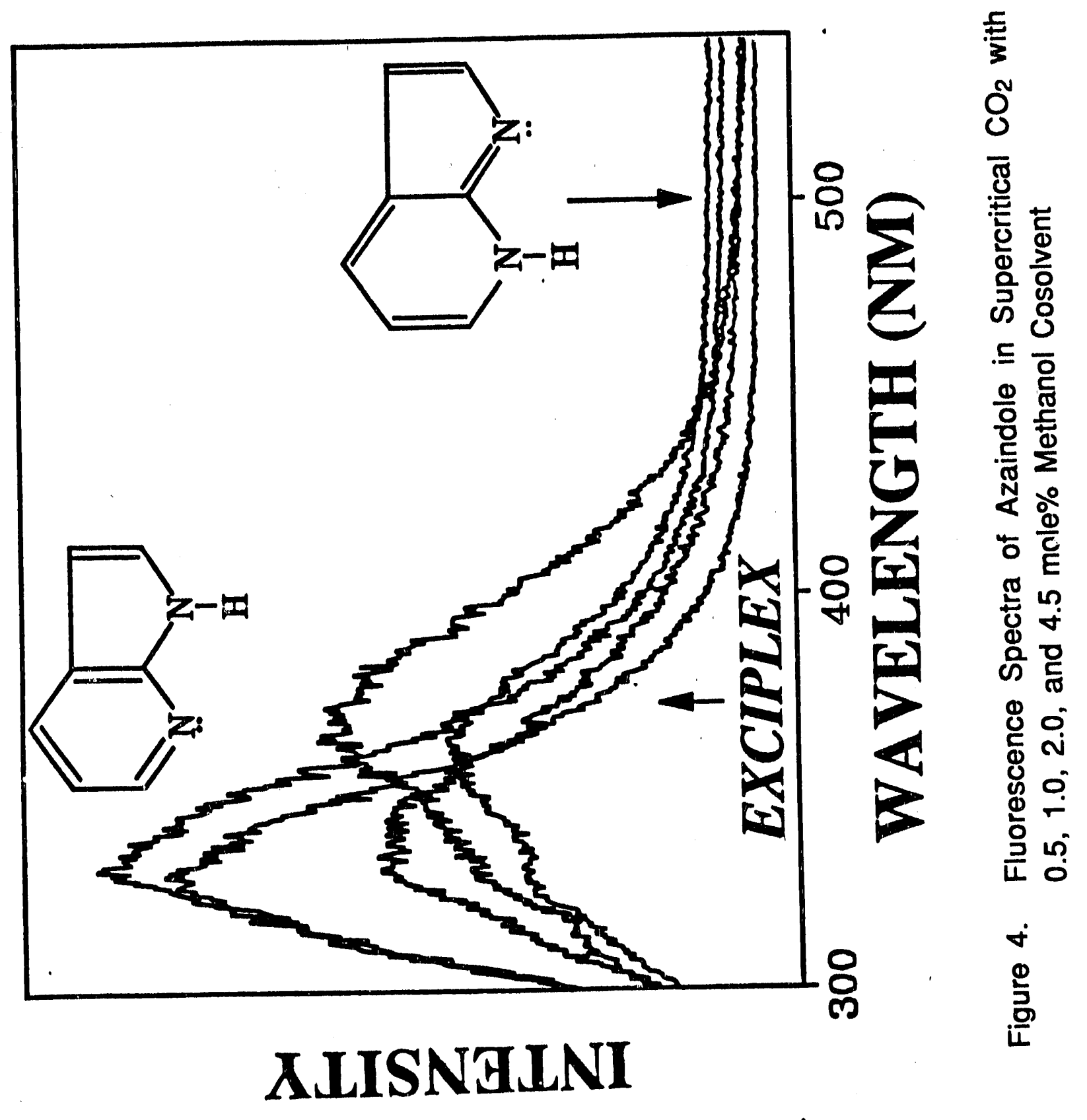



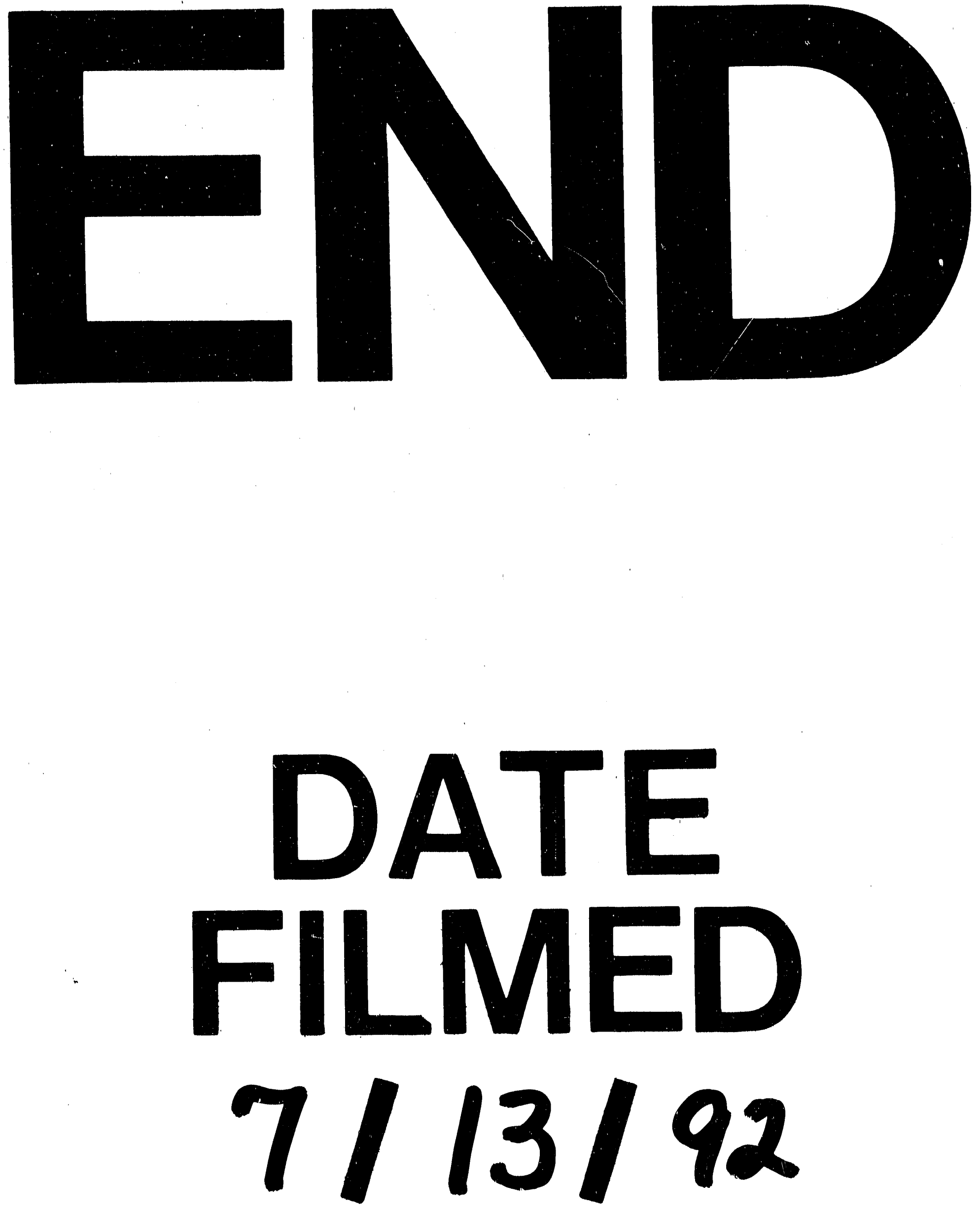
